\title{
Kinetoelastodynamics Modeling and Analysis of Spatial Parallel Mechanism
}

\author{
Xiulong Chen, ${ }^{1}$ Yunfeng Li, ${ }^{1}$ Yu Deng, ${ }^{1}$ Wenbin $\mathrm{Li},{ }^{1}$ and Haibin $\mathrm{Wu}^{2}$ \\ ${ }^{1}$ College of Mechanical and Electronic Engineering, Shandong University of Science and Technology, Qingdao 266590, China \\ ${ }^{2}$ Jiangsu Digital Manufacture Main Laboratory, Huaian 223003, China \\ Correspondence should be addressed to Xiulong Chen; cxldy99@163.com
}

Received 11 February 2015; Revised 13 April 2015; Accepted 15 April 2015

Academic Editor: Tony Murmu

Copyright (C) 2015 Xiulong Chen et al. This is an open access article distributed under the Creative Commons Attribution License, which permits unrestricted use, distribution, and reproduction in any medium, provided the original work is properly cited.

\begin{abstract}
The nonlinear elastodynamic modeling and analysis of the 4-UPS-UPU spatial 5-degree-of-freedom parallel mechanism are investigated. The kinetoelastodynamics theory is used to derive the elastic dynamic equations of 4-UPS-UPU spatial parallel mechanism. In order to grasp the effect of geometric nonlinearity on dynamic behaviors, such as displacement error output, velocity error output, acceleration error output, stress of driving limbs, and natural frequencies, the variations of dynamic behaviors considering geometric nonlinearity and without considering geometric nonlinearity are discussed, respectively. The numerical simulation results show the nonlinear elastodynamic model established can reasonably reflect the dynamic behaviors of 4-UPSUPU spatial parallel mechanism with flexible driving limbs. And geometric nonlinearity is demonstrated to have significant impact on dynamic response and dynamic characteristics of spatial parallel mechanism. The researches can provide important theoretical base for the optimal design of spatial parallel mechanism.
\end{abstract}

\section{Introduction}

The spatial parallel mechanism has a series of advantages, such as high speed, high acceleration, stronger bearing capacity, and higher ratio of stiffness and weight $[1,2]$. At present, the main development trend of spatial parallel mechanism is constantly pursuing high speed, light weight, high precision, and high stability. High speed could enhance working efficiency, high precision could fit the precise job, and light weight could reduce energy consumption. In the high speed working conditions, the light weight driving limbs of spatial parallel mechanism are bound to have a certain degree of elastic deformation, which can cause the motion error and vibration of the mechanism and lead to the decrease of the kinematics performance and dynamics performance of mechanism [3, 4]. Therefore, the elastic deformation of construction members must be considered.

Spatial parallel mechanism is essentially a nonlinear dynamics system with more flexible bodies and rigid bodies. Dynamics modeling and analysis of spatial parallel mechanism were more complex than planar parallel mechanism and serial mechanism. Up to now the research of this aspect is still in the initial stage; the corresponding theory and method of elastodynamic modeling and analysis for spatial parallel mechanism are still not mature. Wang and Mills [5] established dynamics model of planar 3-RRR elastic inside links parallel robot by the use of finite element method and Craig-Bampton theory and analyzed the response of moving platform and vibration of inside links bottom. Piras et al. [6] considered axial flexibility, transverse flexibility of elastic inside links, and axial flexibility of ball screw and obtained the relationship of natural frequency of the robot and position by using Ansys to analyze dynamic behavior of planar 3RRR elastic inside links parallel robot. Kang and Mills [7] derived dynamics model of planar 3-RRR elastic inside links parallel robot by the use of the first kind of Lagrange equation. Zhou et al. [8] derived dynamics model of 3-RRR elastic robot by the use of Ansys theory. Zhang et al. [9] established dynamics model of 3-RRR elastic links parallel robot by the use of hypothesizing modal method, verified the correctness of the theoretical model through the numerical simulation. Jianxin and Yong [10] derived the kinetoelastodynamic equation of Tianfu I type spot welding industrial manipulator by 


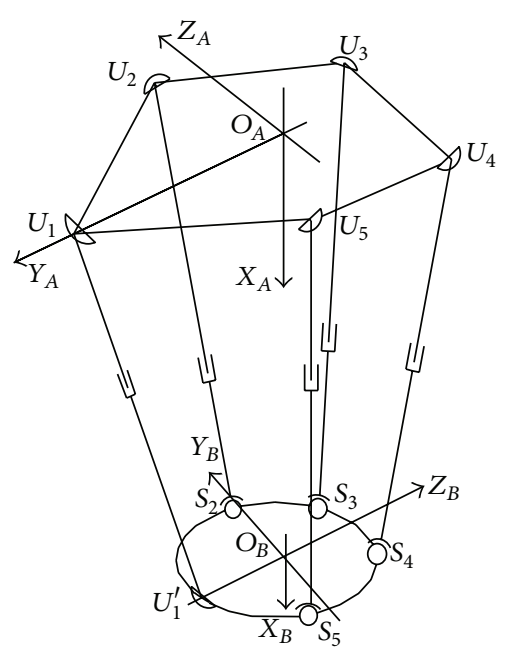

FIGURE 1: Mechanism diagram of 4-UPS-UPU.

the numerical processing technology and obtained natural frequencies and elastic displacement. Minghui and Tian [11] established the elastic dynamic model of diamond parallel manipulator by the use of KED and analyzed natural frequencies of the mechanism. Du et al. [12] derived the dynamical model of flexible parallel robot by using the kinetoelastodynamics theory and Lagrange equation. Shanzeng et al. [13] established the elastic dynamic equations of spatial 3-RRS robot by the use of KED and analyzed the relation of the natural frequencies and design parameters of this mechanism. Li et al. [14] established dynamics and elastodynamics model for 2-DOF planar parallel pick-and-place robot with flexible links. Chen et al. $[15,16]$ studied the elastodynamic behavior of high speed spatial parallel coordinate measuring machines by using flexible multibody dynamics theory and virtual prototyping technology. Zhao et al. $[17,18]$ studied the elastodynamic characteristics of 8-PSS redundant parallel manipulator and 6-PSS parallel manipulator, respectively.

Based on the kinetoelastodynamics theory, the nonlinear elastodynamic model of the 4-UPS-UPU 5-DOF spatial parallel mechanism (see Figure 1), which has three translation degrees of freedom and two rotation degrees of freedom and consists of four UPS (universal joints-prismatic pairsspherical joints) driving limbs, one UPU (universal jointsprismatic pairs-universal joints) driving limb, a fixed platform, and a moving platform, is investigated; the effect of geometric nonlinearity on dynamic behaviors, such as displacement error output, velocity error output, acceleration error output, stress of driving limbs, and natural frequencies, is analyzed.

\section{Kinetoelastodynamics Modeling of Spatial Parallel Mechanism}

The dynamic equations of the 4-UPS-UPU high speed spatial parallel mechanism are built according to the following assumptions.

(1) The moving platform and the stationery platform are considered as rigid body.

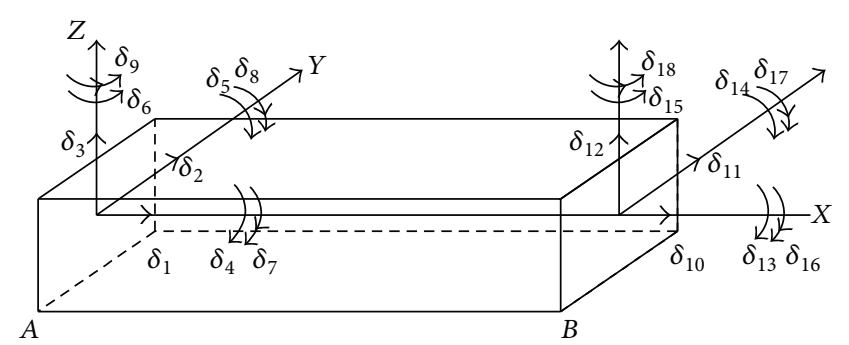

Figure 2: Model of beam element.

(2) The expansion links of driving limbs are considered as elastomers.

2.1. Model of Spatial Beam Element. The rectangle beam element is used to model the spatial parallel mechanism, as shown in Figure 2. Generalized coordinates vectors $\delta=$ $\left[\begin{array}{llll}\delta_{1} & \delta_{2} & \cdots & \delta_{18}\end{array}\right]^{T}$ are introduced to describe the beam element, where $\delta_{1} \sim \delta_{3}$ and $\delta_{10} \sim \delta_{12}, \delta_{4} \sim \delta_{6}$ and $\delta_{13} \sim \delta_{15}$, and $\delta_{7} \sim \delta_{9}$ and $\delta_{16} \sim \delta_{18}$ are the elastic displacement, flexible corner, and curvature of nodes $A$ and $B$, respectively. $N_{A}^{T}$, $N_{B}^{T}, N_{C}^{T}$, and $N_{D}^{T}$ are interpolated vectors, which are decided by the accuracy requirement. $W_{x}(x, t), W_{y}(x, t), W_{z}(x, t)$, $\psi_{x}(x, t), \psi_{y}(x, t)$, and $\psi_{z}(x, t)$ are the functions of elastic displacement along $x, y$, and $z$ and elastic corner around $x$, $y$, and $z$, respectively, which can be written as

$$
\begin{aligned}
& W_{x}(x, t)=N_{A}^{T} \delta, \\
& W_{y}(x, t)=N_{B}^{T} \delta, \\
& W_{z}(x, t)=N_{C}^{T} \delta, \\
& \psi_{x}(x, t)=N_{D}^{T} \delta, \\
& \psi_{y}(x, t)=\dot{N}_{C}^{T} \delta, \\
& \psi_{z}(x, t)=\dot{N}_{B}^{T} \delta .
\end{aligned}
$$

In the moving process of system, the element's elastic displacement is so small that the coupling effect between rigid motion and elastic deformation movement of element can be ignored. That is to say, the absolute velocity of element can be considered as superposition of rigid motion speed and elastic deformation speed, which can be described by the following equation, and the calculation method of the absolute acceleration is similar:

$$
\begin{aligned}
& \dot{W}_{a x}(x, t)=\dot{W}_{r x}(x, t)+\dot{W}_{x}(x, t), \\
& \dot{W}_{a y}(x, t)=\dot{W}_{r y}(x, t)+\dot{W}_{y}(x, t), \\
& \dot{W}_{a z}(x, t)=\dot{W}_{r z}(x, t)+\dot{W}_{z}(x, t), \\
& \dot{\psi}_{a x}(x, t)=\dot{\psi}_{r x}(x, t)+\dot{\psi}_{x}(x, t),
\end{aligned}
$$

where $\dot{W}_{a x}(x, t), \dot{W}_{a y}(x, t)$, and $\dot{W}_{a z}(x, t)$ were the absolute speeds along the $x$-axis, $y$-axis, and $z$-axis, respectively. 
$\dot{W}_{r x}(x, t), \dot{W}_{r y}(x, t)$, and $\dot{W}_{r z}(x, t)$ were the rigid speeds along the $x$-axis, $y$-axis, and $z$-axis, respectively. $\dot{W}_{x}(x, t), \dot{W}_{y}(x, t)$, and $\dot{W}_{z}(x, t)$ were the elastic speeds along the $x$-axis, $y$-axis, and $z$-axis, respectively. $\dot{\psi}_{a x}(x, t), \dot{\psi}_{r x}(x, t)$, and $\dot{\psi}_{x}(x, t)$ were the absolute angular speed, rigid angular speed, and elastic angular speed around the $x$-axis, respectively.

\subsection{Dynamic Equations of Beam Element}

2.2.1. Kinetic Energy of Element. Assuming that the quality of each element's section is concentrated on the axis and the rotational kinetic energy of each element's section is ignored, then the kinetic energy of element without considering the rigid-flexible coupling effect can be written as

$$
\begin{aligned}
T & =\frac{1}{2} \int_{0}^{L} m(x)\left[\left(\frac{d W_{a x}(x, t)}{d t}\right)^{2}+\left(\frac{d W_{a y}(x, t)}{d t}\right)^{2}\right. \\
& \left.+\left(\frac{d W_{a z}(x, t)}{d t}\right)^{2}\right] d x+\frac{1}{2} \int_{0}^{L} \rho I_{p}\left(\frac{d \psi_{a x}(x, t)}{d t}\right)^{2},
\end{aligned}
$$

where $L$ is the length of element. $\rho$ is the density of element. $A$ is the cross-sectional area of element. $I_{p}$ is the polar moment of inertia along the $x$-axis caused by cross section of element. $m(x)$ is the mass distribution function of element.

Equation (3) can be reduced to

$$
T=\frac{1}{2}\left(\dot{\delta}_{r}+\dot{\delta}\right)^{T} M_{e}\left(\dot{\delta}_{r}+\dot{\delta}\right),
$$

where $M_{e}=\rho A \int_{0}^{L} N N^{T} d x$ is the mass matrix of element.

2.2.2. Deformation Energy of the Element. The deformation energy of the element, which includes bending deformation energy, tension/compression deformation energy, and torsion deformation energy, is caused by the bending moment, axial force, and torque of the beam. The total deformation energy of the element considering geometric nonlinearity is given by

$$
\begin{aligned}
V & =\frac{1}{2} E \int_{0}^{L}\left[I_{z}\left(\frac{\partial^{2} W_{y}(x, t)}{\partial x^{2}}\right)^{2}\right. \\
& \left.+I_{y}\left(\frac{\partial^{2} W_{z}(x, t)}{\partial x^{2}}\right)^{2}\right] d x+\frac{1}{2} \\
& \cdot \int_{0}^{L} G I_{p}\left(\frac{\partial \psi_{a x}(x, t)}{\partial x}\right)^{2} d x+\frac{1}{2} E A \int_{0}^{L}\left[\frac{\partial W_{x}(x, t)}{\partial x}\right. \\
& +\frac{1}{2}\left(\frac{\partial W_{x}(x, t)}{\partial x}\right)^{2}+\frac{1}{2}\left(\frac{\partial W_{y}(x, t)}{\partial x}\right)^{2} \\
& \left.+\frac{1}{2}\left(\frac{\partial W_{z}(x, t)}{\partial x}\right)^{2}\right]^{2} d x,
\end{aligned}
$$

where $E$ is tension and compression modulus. $G$ is shear modulus. $I_{y}$ is principal moment of inertia for the $y$-axis of beam element cross section. $I_{z}$ is principal moment of inertia for the $z$-axis of beam element cross section. $I_{p}$ is polar moment of inertia for the $x$-axis of beam element cross section. $(1 / 2)\left(\partial W_{x}(x, t) / \partial x\right)^{2},(1 / 2)\left(\partial W_{y}(x, t) / \partial x\right)^{2}$, and $(1 / 2)\left(\partial W_{z}(x, t) / \partial x\right)^{2}$ are additional tensile and compressive strain caused by a quadratic term; in this session, the additional tensile and compressive strain, which are the geometric nonlinearities factors, have been introduced to the system.

Equation (5) can be reduced to

$$
V=\frac{1}{2} \delta^{T} K_{e 1} \delta,
$$

where

$$
\begin{aligned}
K_{e 1} & =E\left[I_{z} \int_{0}^{L} \ddot{N}_{B} \ddot{N}_{B}^{T} d x+I_{y} \int_{0}^{L} \ddot{N}_{C} \ddot{N}_{C}^{T} d x\right] \\
& +G I_{p} \int_{0}^{L} \dot{N}_{D} \dot{N}_{D}^{T} d x \\
& +E A \int_{0}^{L}\left[\dot{N}_{A}+\frac{1}{2}\left(\dot{N}_{A} \dot{N}_{A}^{T}+\dot{N}_{B} \dot{N}_{B}^{T}+\dot{N}_{C} \dot{N}_{C}^{T}\right) \delta\right] \\
& \cdot\left[N_{A}^{T}+\frac{1}{2} \delta^{T}\left(\dot{N}_{A} \dot{N}_{A}^{T}+\dot{N}_{B} \dot{N}_{B}^{T}+\dot{N}_{C} \dot{N}_{C}^{T}\right)\right] d x
\end{aligned}
$$

is the stiffness matrix of element.

2.2.3. The Elastic Dynamic Equations of Beam Element. Bringing formulas (4) and (6) into Lagrange equation, one obtains

$$
\frac{d}{d t}\left(\frac{\partial T}{\partial \dot{\delta}}\right)-\frac{\partial T}{\partial \delta}+\frac{\partial V}{\partial \delta}=F .
$$

From formula (8), the elastic dynamic equation of element is expressed as

$$
M_{e} \ddot{\delta}+K_{e}^{*} \delta=F_{e}+P_{e}+Q_{e},
$$

where

$$
\begin{aligned}
K_{e}^{*} & =K_{e 1}+\frac{1}{2} \widehat{\delta}^{T} \overline{K_{e 1}} \\
\widehat{\delta}^{T} & =\operatorname{diag}\left(\delta_{1} \cdots \delta_{18}\right) \\
\overline{K_{e 1}} & =\left[\begin{array}{c}
\frac{\partial K_{e 1}}{\partial \delta_{1}} \\
\frac{\partial K_{e 1}}{\partial \delta_{2}} \\
\vdots \\
\frac{\partial K_{e 1}}{\partial \delta_{17}} \\
\frac{\partial K_{e 1}}{\partial \delta_{18}}
\end{array}\right] .
\end{aligned}
$$

$F_{e}$ is generalized force array of element caused by external applied load. $P_{e}$ is force array of research element caused by 
connecting unit. $Q_{e}=-M_{e} \ddot{\delta}_{r}$ is the rigid inertial force array of the system unit.

The generalized coordinates array denoted by $\delta$ in the local coordinate system will be transformed into the system coordinate system (i.e., the stationary coordinate system). Firstly, the unit generalized coordinates array of the system coordinate denoted by $\bar{\delta}_{i j}$ is introduced. Then $\delta=\overline{\mathbf{R}}_{i j} \bar{\delta}_{i j}$, which is the generalized coordinates relational equation, is established. Finally, the dynamic model of element in stationary coordinate system with considering geometric nonlinearity is given by

$$
\bar{M}_{i j} \ddot{\bar{\delta}}_{i j}+\bar{C}_{i j} \dot{\bar{\delta}}_{i j}+\bar{K}_{i j} \bar{\delta}_{i j}=\bar{P}_{i j} \text {, }
$$

where

$$
\begin{aligned}
\bar{M}_{i j} & =\bar{R}_{i j}^{T} M_{e} \bar{R}_{i j}, \\
\bar{C}_{i j} & =2 \bar{R}_{i j}^{T} M_{e} \dot{\bar{R}}_{i j}, \\
\bar{K}_{i j} & =\bar{R}_{i j}^{T} M_{e} \ddot{\bar{R}}_{i j}+\bar{R}_{i j}^{T} K_{e 1}^{*} \bar{R}_{i j}, \\
\bar{P}_{i j} & =\bar{R}_{i j}^{T}\left(F_{e}+P_{e}+Q_{e}\right) .
\end{aligned}
$$

2.3. The Elastic Dynamic Equations of Driving Limbs. According to the finite element method, one of the driving limbs of 4-UPS-UPU parallel mechanism is averagely divided into $m$ beam elements, namely, $1,2, \ldots, n, \ldots, m$, where $n$ represents the sleeve of driving limbs and the telescopic rod of driving limbs contact at the $n$th unit. The figure of the finite element model of each driving limb is shown in Figure 3.

When $j=1,2, \ldots, n-1$, the generalized coordinates of these elements expressed as $\bar{\delta}_{i j}$ are equal to zero because these units are surrounded by a rigid body.

When $j=n, n+1, \ldots, m-1$, the generalized coordinates of the elements in front of nine expressed as $\bar{\delta}_{i n 1}, \bar{\delta}_{i n 2}, \ldots, \bar{\delta}_{i n 9}$ are equal to zero because the left end of the $n$th unit is surrounded by a rigid body.

While the right end of this unit is always coincident at the left end of the next unit, one obtains

$$
\begin{aligned}
& \bar{\delta}_{i j 10}=\bar{\delta}_{i(j+1) 1}, \\
& \bar{\delta}_{i j 11}=\bar{\delta}_{i(j+1) 2}, \\
& \bar{\delta}_{i j 12}=\bar{\delta}_{i(j+1) 3}, \\
& \bar{\delta}_{i j 13}=\bar{\delta}_{i(j+1) 4}, \\
& \bar{\delta}_{i j 14}=\bar{\delta}_{i(j+1) 5}, \\
& \bar{\delta}_{i j 15}=\bar{\delta}_{i(j+1) 6}, \\
& \bar{\delta}_{i j 16}=\bar{\delta}_{i(j+1) 7}, \\
& \bar{\delta}_{i j 17}=\bar{\delta}_{i(j+1) 8}, \\
& \bar{\delta}_{i j 18}=\bar{\delta}_{i(j+1) 9} .
\end{aligned}
$$

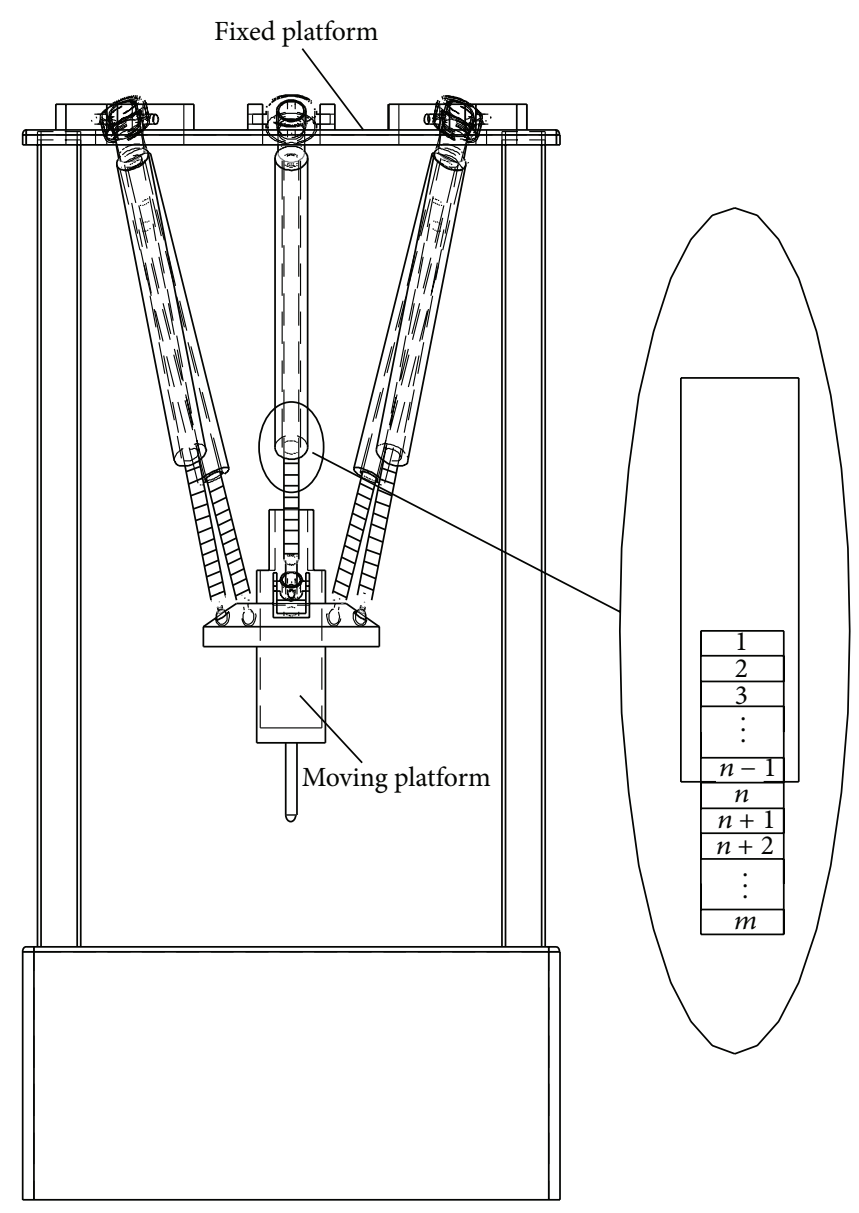

FIGURE 3: Figure of the finite element model of each driving limb.

Considering the constraints of moving platform on the right end point of the $m$ unit, the curvatures of end points on different telescopic rod of driving $\operatorname{limb} i$ are not equal.

When $i=1$, one can get $\bar{\delta}_{i m 17}=\bar{\delta}_{i m 18}=0$.

When $i=2,3,4,5$, on can get $\bar{\delta}_{i m 16}=\bar{\delta}_{i m 17}=\bar{\delta}_{i m 18}=0$.

The generalized coordinates of driving limbs obtained from the above analysis are expressed as follows.

When $i=1$, the number of the generalized coordinates of driving limbs is $9(m-n)+7$; that is,

$$
\begin{aligned}
& {\left[\bar{\delta}_{i(n+1) 1}, \bar{\delta}_{i(n+1) 2}, \ldots, \bar{\delta}_{i(n+1) 9}, \bar{\delta}_{i(n+2) 1}, \ldots, \bar{\delta}_{i m 9}, \ldots,\right.} \\
& \left.\bar{\delta}_{i m 16}\right]^{T}=q_{i} .
\end{aligned}
$$

When $i=2,3,4,5$, the number of the generalized coordinates of driving limbs is $9(m-n)+6$; that is,

$$
\begin{aligned}
& {\left[\bar{\delta}_{i(n+1) 1}, \bar{\delta}_{i(n+1) 2}, \ldots, \bar{\delta}_{i(n+1) 9}, \bar{\delta}_{i(n+2) 1}, \ldots, \bar{\delta}_{i m 9}, \ldots,\right.} \\
& \left.\bar{\delta}_{i m 15}\right]^{T}=q_{i} .
\end{aligned}
$$


The relationship between $q_{i}$ and $\bar{\delta}_{i j}$ is given by

$$
\overline{\boldsymbol{\delta}}_{i j}=\bar{A}_{i j} q_{i}
$$

By taking $\overline{\boldsymbol{\delta}}_{i j}=\bar{A}_{i j} q_{i}$ into (11), we can obtain

$$
\widehat{M}_{i j} \ddot{q}_{i}+\widehat{C}_{i j} \dot{q}_{i}+\widehat{K}_{i j} q_{i}=\widehat{P}_{i j} \text {, }
$$

where

$$
\begin{aligned}
\widehat{M}_{i j} & =\bar{A}_{i j}^{T} \bar{M}_{i j} \bar{A}_{i j}, \\
\widehat{C}_{i j} & =\bar{A}_{i j}^{T} \bar{C}_{i j} \bar{A}_{i j}, \\
\widehat{K}_{i j} & =\bar{A}_{i j}^{T} \bar{K}_{i j} \bar{A}_{i j}, \\
\widehat{P}_{i j} & =\bar{A}_{i j}^{T} \bar{P}_{i j} .
\end{aligned}
$$

Each unit has its elastic dynamic equation like formula (17), so accumulating all the equations, the dynamic model of driving limb $i$ is given by

$$
\bar{M}_{i} \ddot{q}_{i}+\bar{C}_{i} \dot{q}_{i}+\bar{K}_{i} q_{i}=\widetilde{P}_{i},
$$

where

$$
\begin{gathered}
\bar{M}_{i}=\sum_{j=1}^{m} \widehat{M}_{i j}, \\
\bar{C}_{i}=\sum_{j=1}^{m} \widehat{C}_{i j}, \\
\bar{K}_{i}=\sum_{j=1}^{m} \widehat{K}_{i j}, \\
\widetilde{P}_{i}=\sum_{j=1}^{m} \widehat{P}_{i j} .
\end{gathered}
$$

\subsection{Dynamic Equations of Spatial Parallel Mechanism}

2.4.1. Kinematic Constraint Equations. The kinematic constraint equation of 4-UPS-UPU spatial parallel mechanism is expressed as

$$
\mathbf{q}_{S i}=\left[\begin{array}{cccccc}
1 & 0 & 0 & 0 & { }^{A} Z_{S i} & -{ }^{A} Y_{S i} \\
0 & 1 & 0 & -{ }^{A} Z_{S i} & 0 & { }^{A} X_{S i} \\
0 & 0 & 1 & { }^{A} Y_{S i} & -{ }^{A} X_{S i} & 0
\end{array}\right] \mathbf{q}_{0} .
$$

Equation (21) can be reduced to

$$
\mathbf{q}_{S i}=\mathbf{J}_{S i} \mathbf{q}_{0},
$$

where ${ }^{A} X_{S i},{ }^{A} Y_{S i}$, and ${ }^{A} Z_{S i}$ are the coordinates of $S_{i} \cdot \mathbf{q}_{S i}=$ $\left[\begin{array}{lll}\bar{\delta}_{i m 10} & \bar{\delta}_{i m 11} & \bar{\delta}_{i m 12}\end{array}\right]^{T}$ is the elastic displacement vector of $S_{i}$ on $U_{i} P_{i} S_{i}$ driving limb. $\mathbf{q}_{0}$ is the displacement of moving platform caused by elastic deformation. $\mathbf{J}_{S i}$ is the kinematic constraint matrix.
2.4.2. Dynamic Constraint Equations. The dynamic constraint equation of 4-UPS-UPU spatial parallel mechanism is expressed as

$$
\begin{aligned}
& {\left[\begin{array}{cccccc}
m_{0} & 0 & 0 & 0 & 0 & 0 \\
0 & m_{0} & 0 & 0 & 0 & 0 \\
0 & 0 & m_{0} & 0 & 0 & 0 \\
0 & 0 & 0 & I_{x x} & I_{x y} & I_{x z} \\
0 & 0 & 0 & I_{y x} & I_{y y} & I_{y z} \\
0 & 0 & 0 & I_{z x} & I_{z y} I_{z z}
\end{array}\right]\left[\begin{array}{c}
\ddot{X}_{B}+\ddot{q}_{1} \\
\ddot{Y}_{B}+\ddot{q}_{2} \\
\ddot{Z}_{B}+\ddot{q}_{3} \\
\ddot{\gamma}+\ddot{q}_{4} \\
\ddot{\beta}+\ddot{q}_{5} \\
\ddot{\alpha}+\ddot{q}_{6}
\end{array}\right]} \\
& =\left[\begin{array}{l}
\sum F_{i x} \\
\sum F_{i y} \\
\sum F_{i z} \\
\sum M_{i x} \\
\sum M_{i y} \\
\sum M_{i z}
\end{array}\right]+\left[\begin{array}{l}
\sum F_{o x} \\
\sum F_{o y} \\
\sum F_{o z} \\
\sum M_{o x} \\
\sum M_{o y} \\
\sum M_{o z}
\end{array}\right] .
\end{aligned}
$$

After being arranged, (23) can be written as

$\left[\begin{array}{cccccc}m_{0} & 0 & 0 & 0 & 0 & 0 \\ 0 & m_{0} & 0 & 0 & 0 & 0 \\ 0 & 0 & m_{0} & 0 & 0 & 0 \\ 0 & 0 & 0 & I_{x x} & I_{x y} & I_{x z} \\ 0 & 0 & 0 & I_{y x} & I_{y y} & I_{y z} \\ 0 & 0 & 0 & I_{z x} & I_{z y} & I_{z z}\end{array}\right]\left[\begin{array}{l}\ddot{q}_{1} \\ \ddot{q}_{2} \\ \ddot{q}_{3} \\ \ddot{q}_{4} \\ \ddot{q}_{5} \\ \ddot{q}_{6}\end{array}\right]$

$$
=\left[\begin{array}{c}
\sum F_{i x} \\
\sum F_{i y} \\
\sum F_{i z} \\
\sum M_{i x} \\
\sum M_{i y} \\
\sum M_{i z}
\end{array}\right]+\left[\begin{array}{c}
\sum F_{o x} \\
\sum F_{o y} \\
\sum F_{o z} \\
\sum M_{o x} \\
\sum M_{o y} \\
\sum M_{o z}
\end{array}\right]
$$

$$
-\left[\begin{array}{cccccc}
m_{0} & 0 & 0 & 0 & 0 & 0 \\
0 & m_{0} & 0 & 0 & 0 & 0 \\
0 & 0 & m_{0} & 0 & 0 & 0 \\
0 & 0 & 0 & I_{x x} & I_{x y} & I_{x z} \\
0 & 0 & 0 & I_{y x} & I_{y y} & I_{y z} \\
0 & 0 & 0 & I_{z x} & I_{z y} & I_{z z}
\end{array}\right]\left[\begin{array}{c}
\ddot{X}_{B} \\
\ddot{Y}_{B} \\
\ddot{Z}_{B} \\
\ddot{\gamma} \\
\ddot{\beta} \\
\ddot{\alpha}
\end{array}\right] .
$$

Equation (24) can be reduced to

$$
M_{0} \ddot{q}_{0}=f_{0}+F_{0}-M_{0} \ddot{q}_{0 r} .
$$

When defining $Q_{0}=f_{0}+F_{0}-M_{0} \ddot{q}_{0 r}$, (25) is given by

$$
M_{0} \ddot{q}_{0}=Q_{0} .
$$


2.4.3. Dynamic Equations of Spatial Parallel Mechanism. In order to establish dynamic equations of spatial parallel mechanism, we choose $q_{i}^{*}$. When $i=1$, we can get $q_{i}^{*}=$ $\left[\bar{\delta}_{i(n+1) 1}, \bar{\delta}_{i(n+1) 2}, \ldots, \bar{\delta}_{i(n+1) 9}, \bar{\delta}_{i(n+2) 1}, \ldots, \bar{\delta}_{i m 9}, \bar{\delta}_{i m 13}, \bar{\delta}_{i m 14}\right.$, $\left.\bar{\delta}_{i m 15}, \bar{\delta}_{i m 16}, q_{1}, q_{2}, q_{3}, q_{4}, q_{5}, q_{6}\right]^{T}$. When $i=2,3,4,5$, we can get $q_{i}^{*}$ which does not contain $\bar{\delta}_{i m 16}$.

Based on kinematic constraint equations, we can get $q_{i}=$ $R_{i}^{*} q_{i}^{*}(i=1,2, \ldots, 5)$. Then

$$
M_{i} \ddot{q}_{i}^{*}+C_{i} \dot{q}_{i}^{*}+K_{i} q_{i}^{*}=P_{i} \quad(i=1,2, \ldots, 5),
$$

where

$$
\begin{aligned}
M_{i} & =R_{i}^{* T} \bar{M}_{i} R_{i}^{*}, \\
C_{i} & =R_{i}^{* T} \bar{C}_{i} R_{i}^{*}, \\
K_{i} & =R_{i}^{* T} \bar{K}_{i} R_{i}^{*}, \\
P_{i} & =R_{i}^{* T} \bar{P}_{i} .
\end{aligned}
$$

In order to assemble dynamic equations of spatial parallel mechanism, one can get the following.

When $i=1$, we can get

$$
\begin{aligned}
& q_{0 i}=\left[\bar{\delta}_{i(n+1) 1}, \bar{\delta}_{i(n+1) 2}, \ldots, \bar{\delta}_{i(n+1) 9}, \bar{\delta}_{i(n+2) 1}, \ldots, \bar{\delta}_{i m 9},\right. \\
&\left.\bar{\delta}_{i m 13}, \bar{\delta}_{i m 14}, \bar{\delta}_{i m 15}, \bar{\delta}_{i m 16}\right]^{T} .
\end{aligned}
$$

When $i=2,3,4,5$, we can get

$$
\begin{gathered}
q_{0 i}=\left[\bar{\delta}_{i(n+1) 1}, \bar{\delta}_{i(n+1) 2}, \ldots, \bar{\delta}_{i(n+1) 9}, \bar{\delta}_{i(n+2) 1}, \ldots, \bar{\delta}_{i m 9},\right. \\
\left.\bar{\delta}_{i m 13}, \bar{\delta}_{i m 14}, \bar{\delta}_{i m 15}\right]^{T} .
\end{gathered}
$$

When knowing $q_{0}=\left[q_{1}, q_{2}, q_{3}, q_{4}, q_{5}, q_{6}\right]^{T}, q_{i}^{*}$ can be expressed as

$$
q_{i}^{*}=\left[\begin{array}{l}
q_{0 i} \\
q_{0}
\end{array}\right] .
$$

The generalized coordinate of spatial parallel mechanism is given by

$$
q=\left[\begin{array}{llllll}
q_{01}^{T} & q_{02}^{T} & q_{03}^{T} & q_{04}^{T} & q_{05}^{T} & q_{0}^{T}
\end{array}\right]^{T} .
$$

The generalized coordinate of $\mathbf{q}_{i}^{*}$ on driving limb and $\mathbf{q}$ on system is given by

$$
q_{i}^{*}=R_{i} q
$$

The displacement of moving platform defined as $q_{0}$ and the generalized coordinate defined as $q$ is given by

$$
q_{0}=R_{0} q
$$

When assembling (22) and (26), the dynamic equation of spatial parallel mechanism is written as

$$
\mathbf{M} \ddot{\mathbf{q}}+\widehat{\mathbf{C}} \dot{\mathbf{q}}+\mathbf{K q}=\mathbf{Q}
$$

where $\mathbf{M}=\sum_{i=1}^{5} \mathbf{R}_{i}^{T} \mathbf{M}_{i} \mathbf{R}_{i}+\mathbf{R}_{0}^{T} \mathbf{M}_{0} \mathbf{R}_{0}$ is the mass matrix of system. $\widehat{\mathbf{C}}=\sum_{i=1}^{5} \mathbf{R}_{i}^{T} \mathbf{C}_{i} \mathbf{R}_{i}$ is the gross damping matrix of the system. $\mathbf{K}=\sum_{i=1}^{5} \mathbf{R}_{i}^{T} \mathbf{K}_{i} \mathbf{R}_{i}$ is the stiffness matrix. $\mathbf{Q}=$ $\sum_{i=1}^{5} R_{i}^{T} P_{i}+R_{0}^{T} Q_{0}$ is the generalized force matrix. $\mathbf{q}$ is the generalized coordinate of system.

\section{Kinetoelastodynamics Numerical Calculation and Analysis of Spatial Parallel Mechanism}

3.1. Parameters of 4-UPS-UPU Spatial Parallel Mechanism. The parameters of 4-UPS-UPU spatial parallel mechanism are expressed as follows. The distance between the first universal joint on fixed platform and stationary platform center is $780 \mathrm{~mm}$, the other universal joints on stationary platform are distributed in a circle with a radius of $720 \mathrm{~mm}$, and the hinges on moving platform are distributed in a circle with a radius of $200 \mathrm{~mm}$. The material of driving limbs is steel, density is $7.801 \times 10^{3} \mathrm{~kg} / \mathrm{m}^{3}$, tension and compression modulus of elasticity are $E=2.0 \times 10^{11} \mathrm{~Pa}$, elastic shear modulus is $G=8.0 \times 10^{10} \mathrm{~Pa}$, and Poisson ratio is 0.29 . The mass of moving platform is $m_{0}=36.64 \mathrm{~kg}$ and the moment of inertia of moving platform is $I_{x x}=0.948 \mathrm{~kg} \cdot \mathrm{m}^{2}, I_{x y}=0.81 \times$ $10^{-8} \mathrm{~kg} \cdot \mathrm{m}^{2}, I_{x z}=0.56 \times 10^{-2} \mathrm{~kg} \cdot \mathrm{m}^{2}, I_{y y}=0.66424 \mathrm{~kg} \cdot \mathrm{m}^{2}$, $I_{y z}=1.166 \times 10^{-7} \mathrm{~kg} \cdot \mathrm{m}^{2}, I_{z x}=0.56 \times 10^{-2} \mathrm{~kg} \cdot \mathrm{m}^{2}, I_{z y}=1.166 \times$ $10^{-7} \mathrm{~kg} \cdot \mathrm{m}^{2}$, and $I_{z z}=0.64 \mathrm{~kg} \cdot \mathrm{m}^{2}$. The principal moment of inertia for the $x$-axis, $y$-axis, and $z$-axis of expansion link cross section on driving limbs is $I_{x}=2.512 \times 10^{-7} \mathrm{~m}^{4}, I_{y}=$ $1.256 \times 10^{-7} \mathrm{~m}^{4}$, and $I_{z}=1.256 \times 10^{-7} \mathrm{~m}^{4}$. The length of oscillating rod on driving limbs is $0.76 \mathrm{~m}$ and the length of expansion link on driving limbs is $0.88 \mathrm{~m}$.

3.2. Numerical Calculation and Analysis. Based on nonlinear elastic dynamics equation (35) of 4-UPS-UPU spatial parallel mechanism, the dynamic behaviors, which consist of kinematic error output, stress of driving limbs, and natural frequencies, of parallel mechanism have been studied by numerical simulation. The movement of 4-UPS-UPU parallel mechanism is defined as (unit: $\mathrm{s}, \mathrm{m}$ )

$$
\begin{aligned}
& X=0.95+0.09 t, \\
& Y=-0.15 \cos (\pi t), \\
& Z=0.15 \sin (\pi t), \\
& \alpha=0, \\
& \beta=0,
\end{aligned}
$$

3.2.1. Kinematic Error Output Response. In order to master the influence of geometric nonlinearity on kinematic error output response of parallel mechanism, the kinematic error 


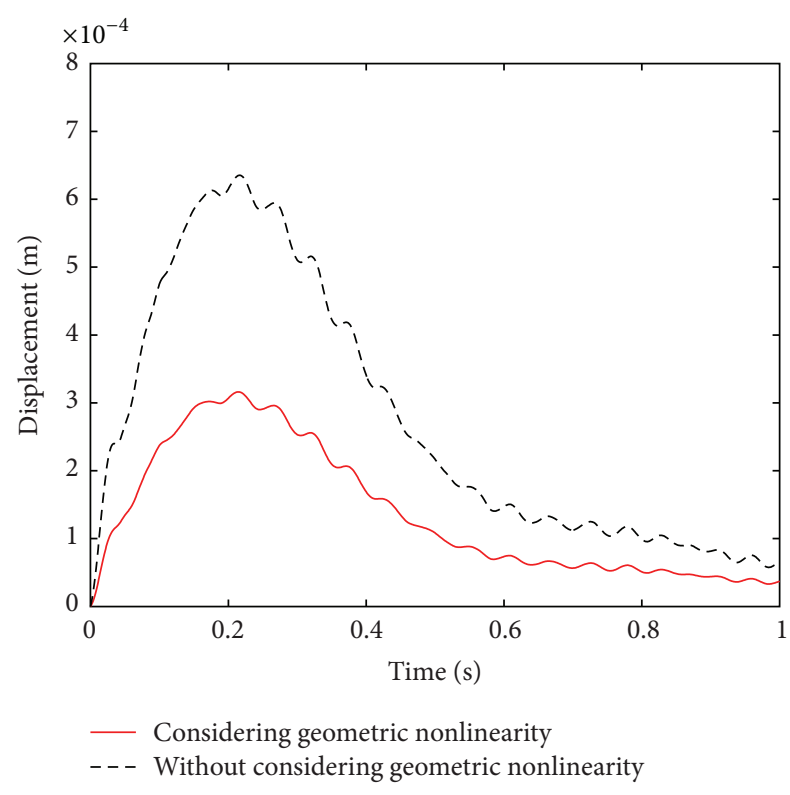

FIgURE 4: Displacement error output of moving platform.

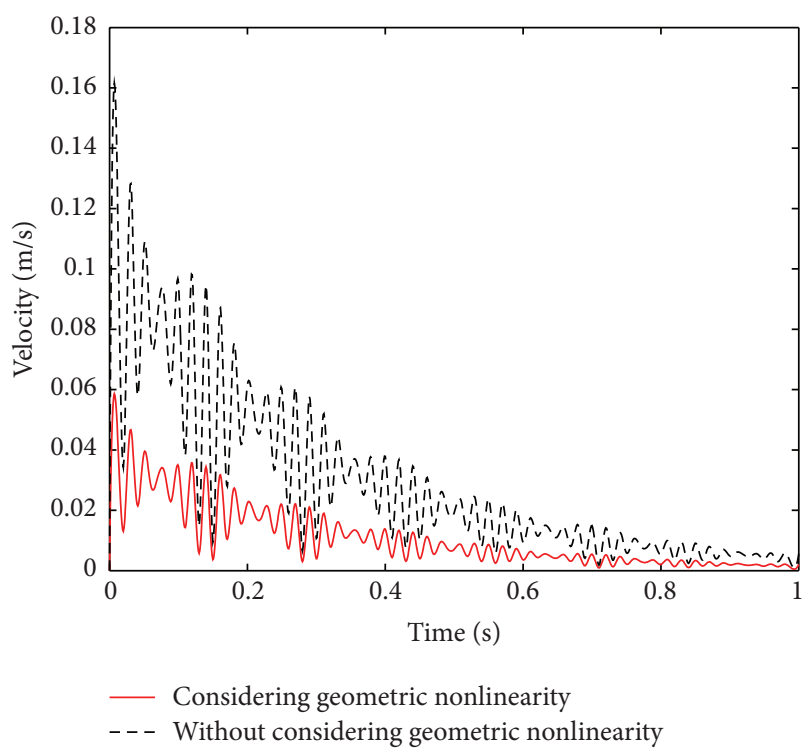

FIGURE 5: Velocity error output of moving platform.

output considering geometry nonlinearity and without considering geometric nonlinearity is analyzed. The displacement error output, velocity error output, and acceleration error output are shown in Figures 4 to 7.

From Figures 4 to 7 , the geometric nonlinearity has significant impact on kinematic error output response including displacement error, velocity error, and acceleration error of parallel mechanism; the value of kinematic error output response considering geometry nonlinearity is obviously less than the value without considering geometric nonlinearity. The displacement error output response fluctuates with

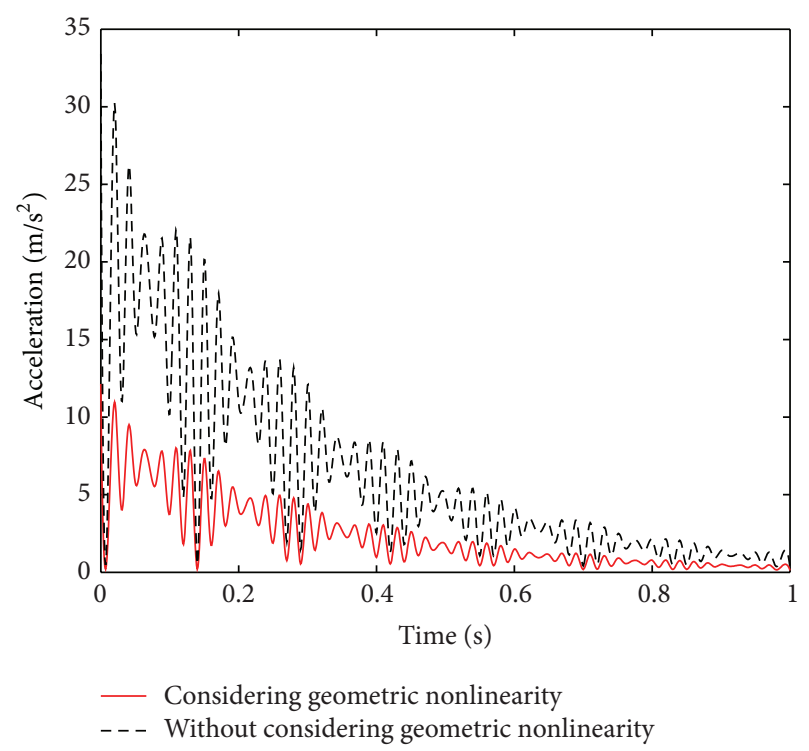

FigURE 6: Acceleration error output of moving platform.

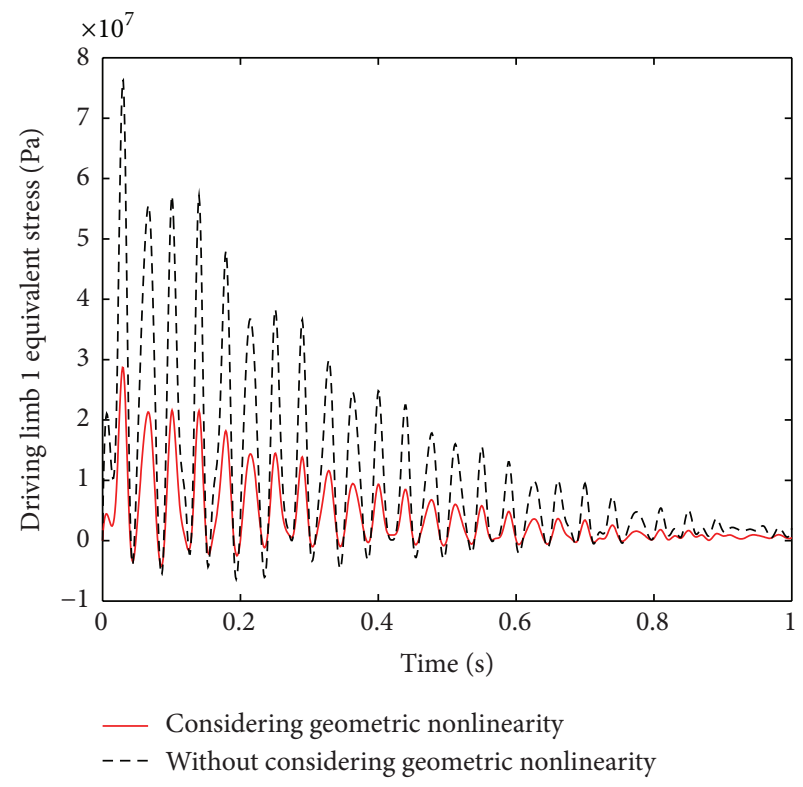

FIGURE 7: Equivalent stress of driving limb 1.

the position of moving platform, and the maximum displacement error is $6.41 \times 10^{-4} \mathrm{~m}$; in the meantime the violent oscillation of velocity error and acceleration error occurs.

3.2.2. Equivalent Stress of Driving Limbs. The maximum equivalent stress of 4-UPS-UPU high speed spatial parallel mechanism appears on the elements which are located at the rear of each driving limb that contacts with the moving platform. In order to master the influence of geometric nonlinearity on equivalent stress of driving limbs, the maximum equivalent stress considering geometry nonlinearity and without considering geometric nonlinearity is analyzed. The maximum equivalent stress of driving limbs is shown in 


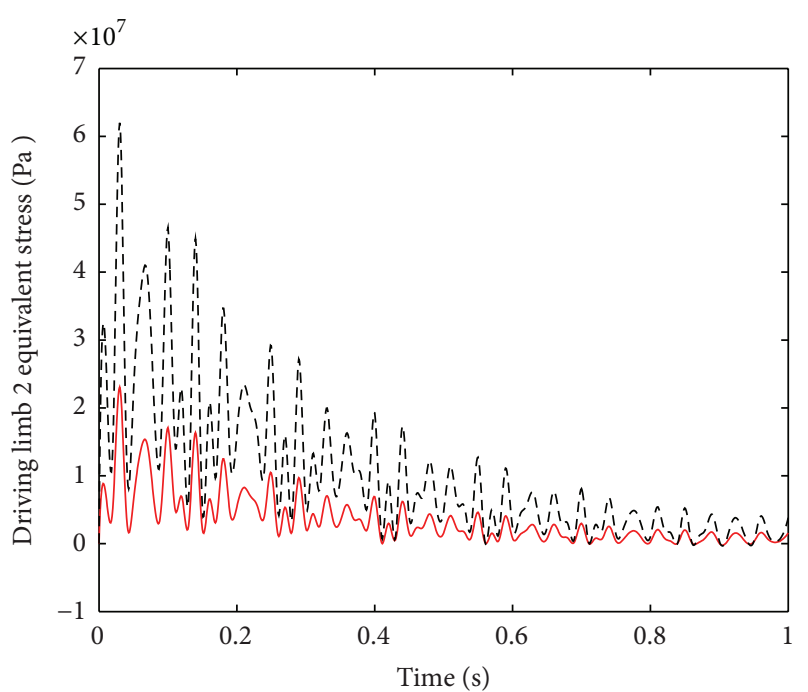

- Considering geometric nonlinearity

- - Without considering geometric nonlinearity

FIGURE 8: Equivalent stress of driving limb 2.

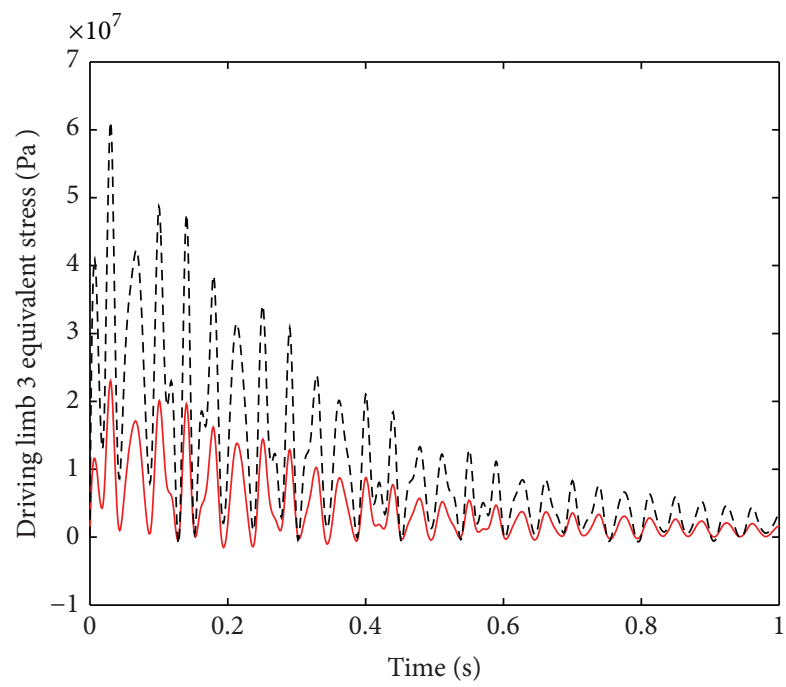

_ Considering geometric nonlinearity

- - - Without considering geometric nonlinearity

FIGURE 9: Equivalent stress of driving limb 3.

Figures 8 to 11. From Figure 1, the UPU limb of 4-UPS-UPU parallel mechanism is defined as driving limb 1 , and driving limb 2, driving limb 3, driving limb 4, and driving limb 5 are distributed clockwise, respectively.

From Figures 8 to 11, geometric nonlinearity has a significant impact on the equivalent stress of driving limbs; the value of equivalent stress without considering geometric nonlinearity is bigger than that considering geometric nonlinearity. The equivalent stress of the driving limbs fluctuates violently throughout the course of the movement, and the values of equivalent stress vary greatly. Comparing the

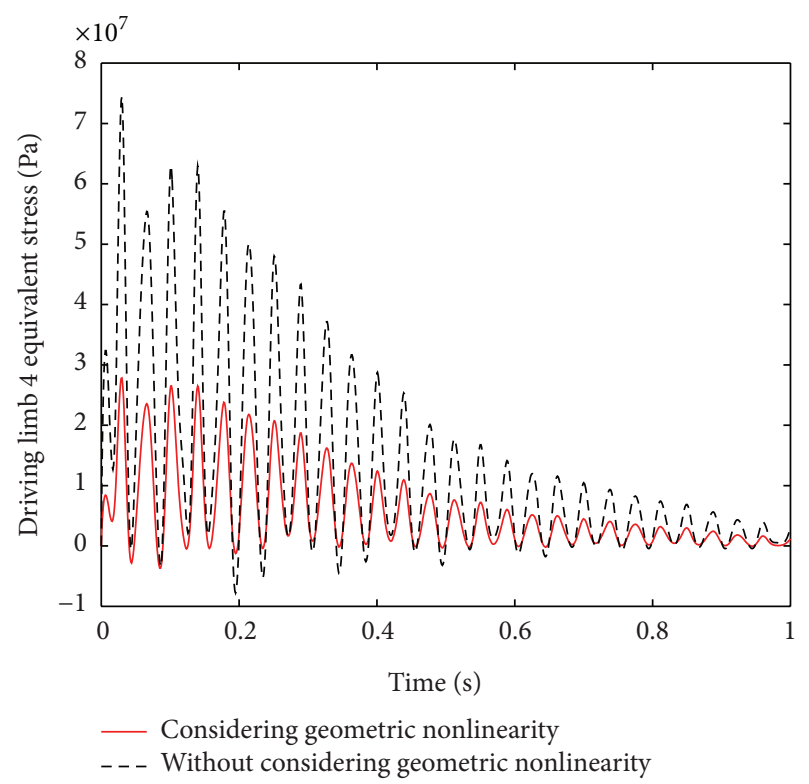

FIgUre 10: Equivalent stress of driving limb 4.

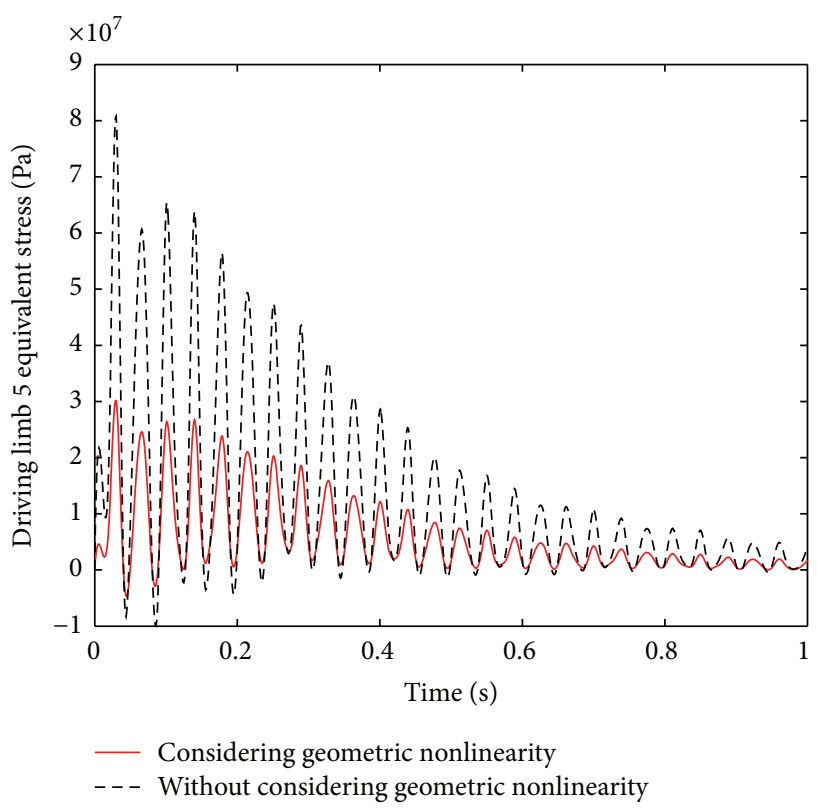

FIGURE 11: Equivalent stress of driving limb 5.

equivalent stress of five driving limbs, the maximum equivalent stress of parallel mechanism appears on driving limb 5.

3.2.3. The Natural Frequency of the System. From the dynamic equations of spatial parallel mechanism $(\mathbf{M} \ddot{\mathbf{q}}+\widehat{\mathbf{C}} \dot{\mathbf{q}}+\mathbf{K q}=$ $\mathbf{Q}(35))$, the characteristic equation of the system can be obtained as

$$
\left|K^{-1} M-\lambda I\right|=0
$$




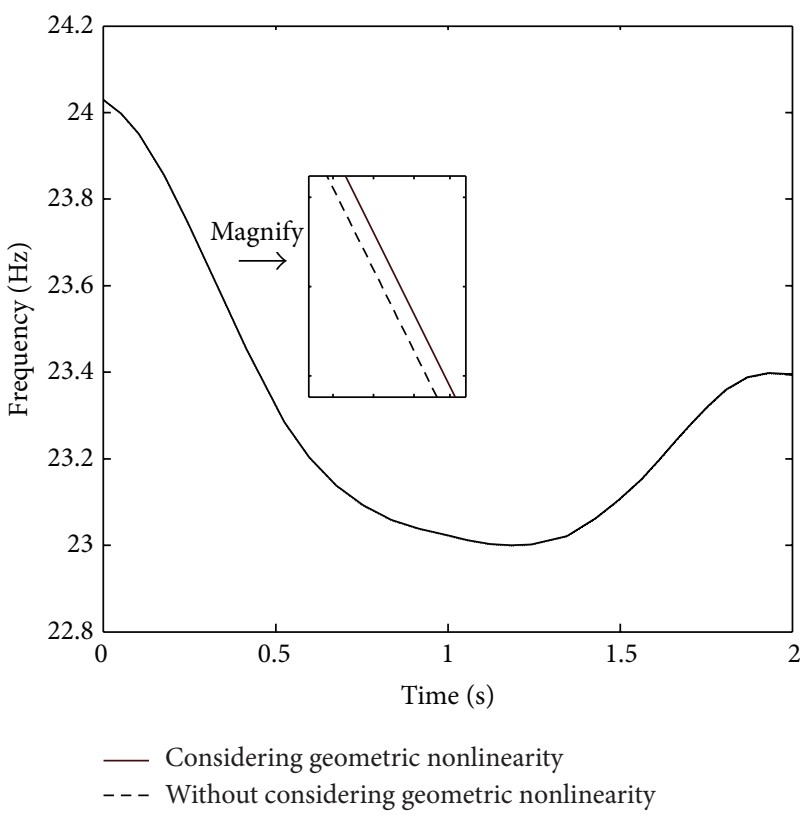

FIGURE 12: Base frequency of 4-UPS-UPU parallel mechanism.

$$
\begin{aligned}
\lambda & =\frac{1}{\omega^{2}}, \\
f_{j} & =\frac{\omega_{j}}{2 \pi}=\frac{\sqrt{(1 / \lambda)}}{2 \pi},
\end{aligned}
$$

where $f_{j}$ is the natural frequency of the system. Sort $f_{j}$ in ascending order; in particular, $f_{1}$ is the base frequency, which is a significant value in effecting the system's dynamic characteristic.

In order to master the influence of geometric nonlinearity on natural frequency of parallel mechanism, the variations of natural frequency considering geometric nonlinearity and without considering geometric nonlinearity were analyzed, respectively, as shown in Figure 12.

According to Figure 12, geometric nonlinearity has a certain influence on natural frequency of the system, the natural frequency fluctuates with position of parallel mechanism, and the curve of natural frequency is smooth. The change amplitude of natural frequency is between 22.8 and $24.2 \mathrm{~Hz}$. As is known to all, the natural frequencies of parallel mechanism are essentially decided by the stiffness matrix and mass matrix of the mechanism. Since the stiffness matrix of elements with considering geometric nonlinearity is different from the stiffness matrix of elements without considering geometric nonlinearity, the stiffness matrices of 4-UPS-UPU spatial parallel mechanism with considering geometric nonlinearity and without considering geometric nonlinearity are not the same, and then the natural frequencies of the mechanism with considering geometric nonlinearity and without considering geometric nonlinearity are unequal. From the above analysis, the geometric nonlinearity can affect the natural frequency of the system to a certain degree.

\section{Conclusion}

(1) Based on kinetoelastodynamics theory, the elastic dynamic equations of 4-UPS-UPU spatial parallel mechanism are established.

(2) The dynamic behavior including the kinematic error output response, the stress of driving limbs, and the system natural frequency is analyzed by using the numerical calculation method, and the variations of considering geometric nonlinearity and without considering geometric nonlinearity are discussed, respectively.

(3) The simulation results not only reflect the dynamic behavior of 4-UPS-UPU spatial parallel mechanism, but also show that the effect of geometric nonlinearity on the dynamic behavior cannot be ignored. The research can give enough attention to the geometric nonlinearity in the resonance analysis, dynamic design, kinematic error analysis, and strength calculation of parallel mechanism.

\section{Conflict of Interests}

The authors declare that there is no conflict of interests regarding the publication of this paper.

\section{Acknowledgments}

This research is supported by the National Natural Science Foundation of China (Grants nos. 51005138, 51375282, and 11272190), Shandong Young Scientists Award Fund (Grant no. BS2012ZZ008), the Science Foundation of SUST (Grant no. 2011KYJQ102), and the project of Jiangsu Key Laboratory of Digital Manufacturing Technology (Grant no. HGDML1104).

\section{References}

[1] O. Piccin, B. Bayle, B. Maurin, and M. de Mathelin, "Kinematic modeling of a 5-DOF parallel mechanism for semi-spherical workspace," Mechanism and Machine Theory, vol. 44, no. 8, pp. 1485-1496, 2009.

[2] X. Chen and X. Sun, "Dexterity analysis of a 4-UPS-RPS parallel mechanism," International Journal of Advanced Robotic Systems, vol. 9, article 144, 2012.

[3] J. Hu and X. Zhang, "Active vibration control and its simulation of a novel 2-DoF flexible parallel manipulator," China Mechanical Engineering, vol. 21, no. 17, pp. 2017-2024, 2010 (Chinese).

[4] S. K. Dwivedy and P. Eberhard, "Dynamic analysis of flexible manipulators, a literature review," Mechanism and Machine Theory, vol. 41, no. 7, pp. 749-777, 2006.

[5] X. Wang and J. K. Mills, "Dynamic modeling of a flexiblelink planar parallel platform using a substructuring approach," Mechanism and Machine Theory, vol. 41, no. 6, pp. 671-687, 2006.

[6] G. Piras, W. L. Cleghorn, and J. K. Mills, "Dynamic finiteelement analysis of a planar high-speed, high-precision parallel manipulator with flexible links," Mechanism and Machine Theory, vol. 40, no. 7, pp. 849-862, 2005. 
[7] B. Kang and J. K. Mills, "Dynamic modeling of structurallyflexible planar parallel manipulator," Robotica, vol. 20, no. 3, pp. 329-339, 2002.

[8] Z. Zhou, J. Xi, and C. K. Mechefske, "Modeling of a fully flexible 3PRS manipulator for vibration analysis," Journal of Mechanical Design, vol. 128, no. 2, pp. 403-412, 2006.

[9] X. Zhang, J. K. Mills, and W. L. Cleghorn, "Dynamic modeling and experimental validation of a 3-PRR parallel manipulator with flexible intermediate links," Journal of Intelligent \& Robotic Systems, vol. 50, no. 4, pp. 323-340, 2007.

[10] Y. Jianxin and C. Yong, "Kineto-elastodynamic analysis of parallel industrial robotic manipulators," Robot, vol. 18, no. 6, pp. 328-331, 1996 (Chinese).

[11] Z. Minghui and H. Tian, "Research on elastic dynamic analysis and test for Diamond mechanism," Journal of Machine Design, vol. 21, no. 11, pp. 6-8, 2004 (Chinese).

[12] Z. Du, Y. Yu, and X. Zhang, "Dynamic modeling of planar flexible parallel manipulators," Chinese Journal of Mechanical Engineering, vol. 43, no. 9, pp. 96-101, 2007 (Chinese).

[13] L. Shanzeng, Y. Yueqing, S. Liying, and Y. Jianxin, "Dynamics modeling and frequency analysis of a 3-RRS flexible parallel manipulator," China Mechanical Engineering, vol. 19, no. 10, pp. 1219-1223, 2008 (Chinese).

[14] H. Li, Z. Yang, and T. Huang, "Dynamics and elasto-dynamics optimization of a 2-DOF planar parallel pick-and-place robot with flexible links," Structural and Multidisciplinary Optimization, vol. 38, no. 2, pp. 195-204, 2009.

[15] X. Chen, D. Wei, W. Li, and Y. Deng, "Nonlinear elastodynamic behaviour analysis of high-speed spatial parallel coordinate measuring machines," International Journal of Advanced Robotic Systems, vol. 9, article 140, 6 pages, 2012.

[16] X.-L. Chen, S.-S. Jia, Y. Deng, and Y.-S. Zhao, "Dynamic behaviors of rigid-flexible coupling for novel 4-UPS-UPU parallel coordinate measuring machine," Journal of Jilin University, vol. 41, no. 4, pp. 1020-1024, 2011 (Chinese).

[17] Y. Zhao, F. Gao, X. Dong, and X. Zhao, "Elastodynamic characteristics comparison of the 8-PSS redundant parallel manipulator and its non-redundant counterpart-the 6-PSS parallel manipulator," Mechanism and Machine Theory, vol. 45, no. 2, pp. 291-303, 2010.

[18] Y. Zhao, F. Gao, X. Dong, and X. Zhao, "Dynamics analysis and characteristics of the 8-PSS flexible redundant parallel manipulator," Robotics and Computer-Integrated Manufacturing, vol. 27, no. 5, pp. 918-928, 2011. 

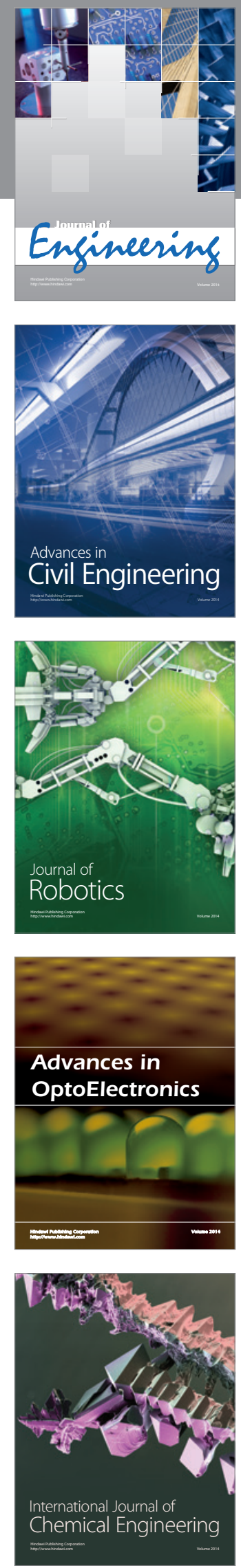

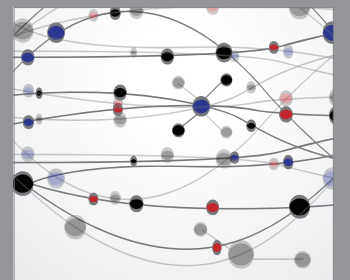

The Scientific World Journal
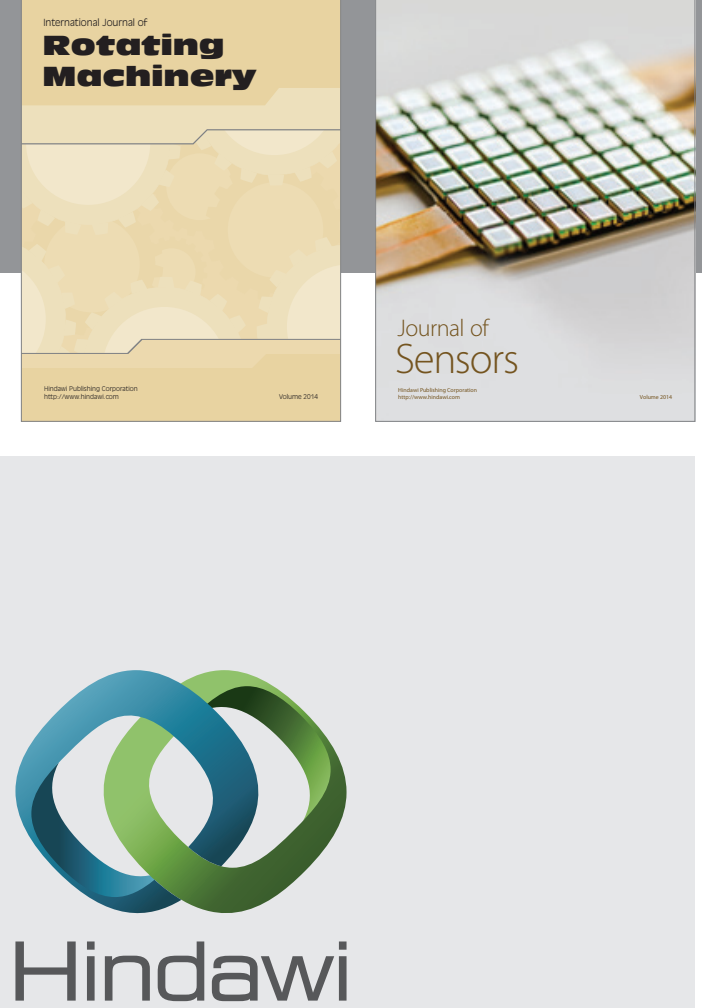

Submit your manuscripts at http://www.hindawi.com
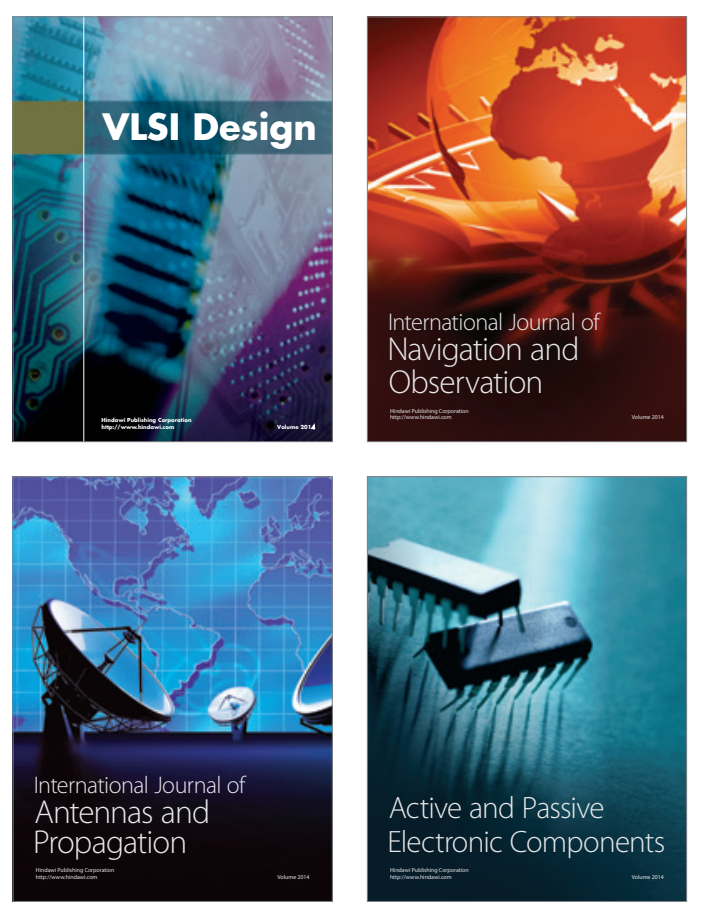
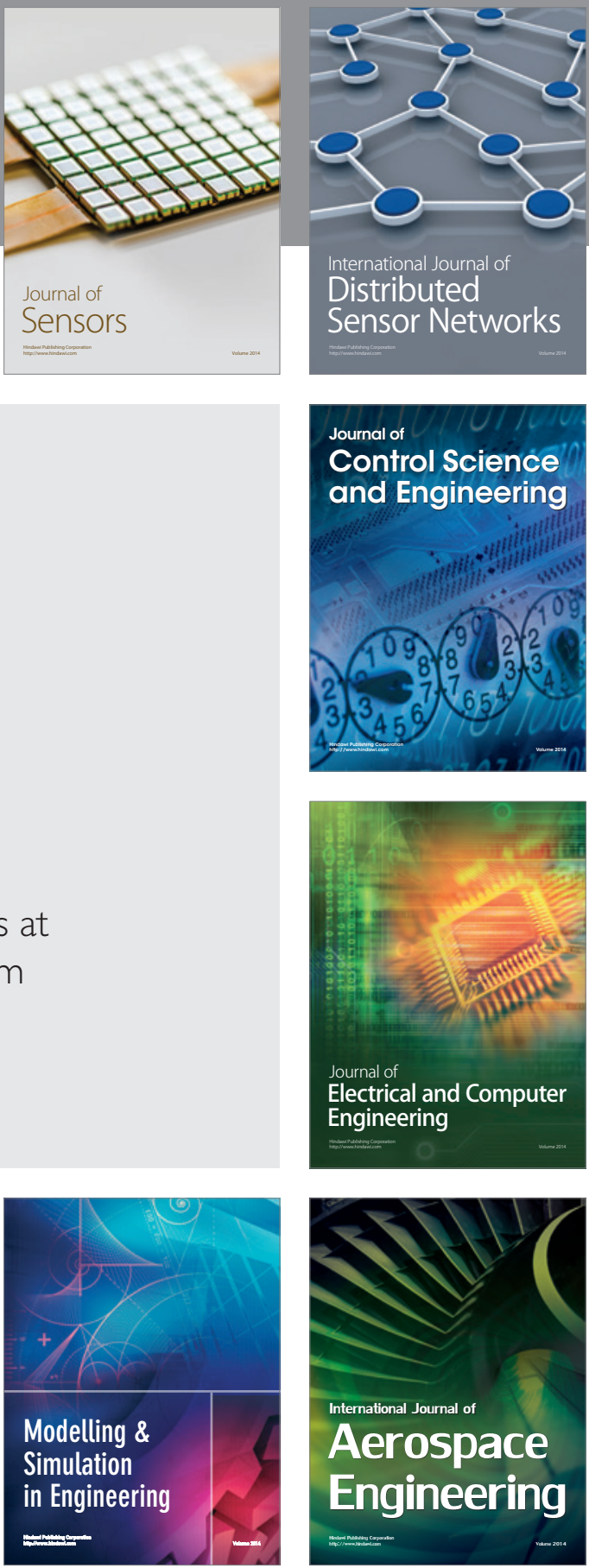

Journal of

Control Science

and Engineering
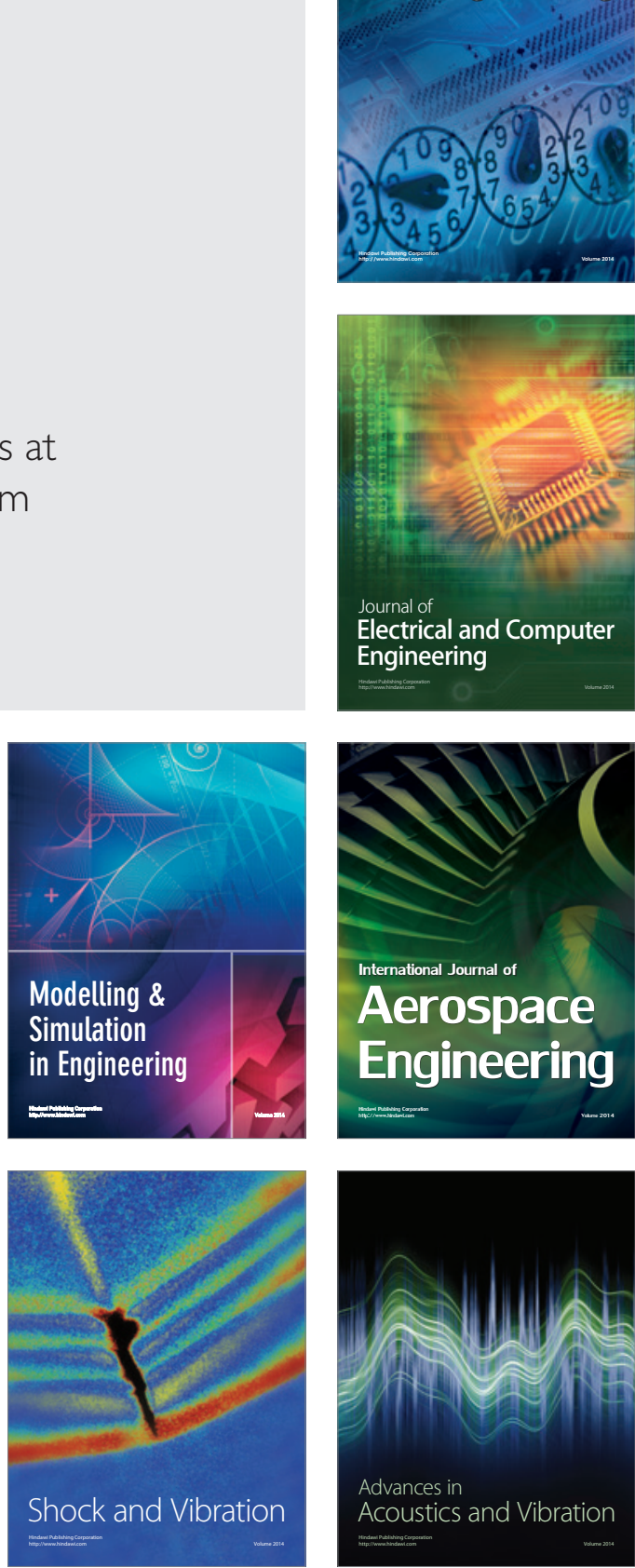